\title{
Identificação Automática de Expertise Analisando Currículos no Formato Lattes
}

Borges ${ }^{1}$, Luiz Carlos Ribeiro Junior ${ }^{1}$, Gustavo Piltcher ${ }^{1}$ Stanley Loh ${ }^{1,2}$, Thyago Bornow ${ }^{1}$, Rodrigo Branco Kickhöfel ${ }^{1}$, Cleber Gouvea ${ }^{1}$, Ramiro Saldaña ${ }^{1}$ Daniel Lichtow

Rua Felix da Cunha, 402 - 96.010-000 - Pelotas - RS - Brasi

Informática - Universidade Luterana do Brasil (ULBRA) Rua Miguel Tostes, 101 , Canoas, RS, Brasil

thyago, 1cr, lichtnow, rodrigok, cgouvea, Isaldana @at.las.ucpel.tche.br sloh@terra.com.br ; gustavopil@gmail.com

Abstract. This paper presents a software system that automatically identifies expertise in personal curriculums, stored in the Lattes format. The identification is made through the extraction of textual information from XML structures used in the Lattes format. Text mining techniques are used to classify the texts according to themes defined in a domain ontology. This process allows identifying user's expertise, that is, competences and areas of interest. Since curriculums in Lattes format are structured by sections (publications, experience, projects, etc), it is possible to identify different areas in different fields of action.

Resumo. Este artigo descreve o desenvolvimento de uma ferramenta para a identificação automática da expertise em curriculos (curriculo vitae) no formato Lattes. Esta identificação se dá através da extração de informações do arquivo XML gerado pela Plataforma Lattes. Uma vez feita à extração, são utilizadas técnicas de Text Mining de forma a fazer a associação dos dados extraidos a uma ontologia de domínio para a identificacão das áreas de atuação e expertise dos usuários. Este trabalho esta sendo desenvolvido para dar auxilio ao Sistema de Recomendação para Apoio à Colaboração (SisRecCol), desenvolvido pelo GPSI (Grupo de Pesquisa em Sistemas de Informação) da UCPel.

1. Introdução

Para que uma organização possa tornar-se mais competitiva dentro do seu ramo de atividade é necessário que ela conheça o perfil de seus funcionários e também dos candidatos a ingressar nela. Para isso, faz-se imprescindivel conhecer as competências destas pessoas para uma melhor avaliação de seus potenciais.

Convém ressaltar que com a velocidade com que surgem novas tecnologias e a constante reformulação de conhecimentos já consolidados, torna-se necessário uma maior agilidade na atualização do conhecimento acerca das competências dos colaboradores de uma organização. 
A organização deve manter uma base de dados sobre as pessoas e suas áreas de atuação ou conhecimento, que funcione como um Mapa de Conhecimento Pages). Estes mapas não armazenam a solução de problemas, mas indicam quem dellow da comunidade possui determinado tipo de conhecimento, estando virtualmente aptro auxiliar na solução de algum problema (Davenport \& Pruzac, 1997).

Neste sentido, definir o perfil de um membro de uma organização, reconhec suas preferências, suas áreas de interesse é uma tarefa importante. Uma forma de estas informações é mediante a aplicação de um questionário a ser preenchido pelo membro da organização

Ocorre, porém que o preenchimento dos dados solicitados no questionário pod não ser preciso. Além disto, é necessário levar em consideração o fato de que o perfil os interesses de uma pessoa são dinâmicos. Assim, as informações sobre o usurí podem ficar obsoletas rapidamente, sendo necessário que sejam feitas atualizações destas informações sempre que o membro da organização começar a trabalhar em uma nova área ou quando ele aperfeiçoar seus conhecimentos.

Para minimizar este problema torna-se interessante à identificação e atualização automática do perfil e dos interesses dos usuários. É importante que esta identificacão possa ser feita a partir de uma fonte de dados que o usuário costume manter atualizada.

Este artigo apresenta um software que tem por objetivo auxiliar na identificação das expertises dos usuários, bem como analisar as competências de um grupo. A ferramenta utiliza o arquivo XML gerado pelo Currículo Lattes desenvolvido pelo Conselho Nacional de Desenvolvimento Científico e Tecnológico (CNPq, 2004).

O presente trabalho está sendo desenvolvido para dar suporte a um Sistema de Recomendação para Apoio à Colaboração (SisRecCol), desenvolvido pelo Grupo de Pesquisa em Sistema de Informações - GPSI (http://gpsi.ucpel.tche.br). Este sistem identifica os assuntos discutidos em seções de um web chat privado e então recomenda itens de uma Biblioteca Digital, discussões anteriores e autoridades no assunto (pessoas que possuem conhecimento mais significativo no assunto tratado), podendo assim ajudar as organizações na Gestão de Competências.

A seção 2 apresenta alguns trabalhos correlatos. A seção 3 discute a extração dos dados utilizando arquivos XML dos currículos do formato Lattes. A seção 4 descreve as técnicas de Text Mining e a utilização de uma ontologia desenvolvida para identificar a expertises dos usuários. Na seção 5 são mostrados experimentos da extração de informações de um Currículo Lattes e testes com artigos científicos para identificar 0 grau de confiabilidade do módulo de Text Mining. O artigo termina com a seção 6 apresentando as conclusões e trabalhos futuros.

\section{Trabalhos Correlatos}

Yimam-Seid e outros (2003) apresentam trabalhos que criam bases de dados das competências dos usuários. Dentre as ferramentas apresentadas está o Expert/ExpertLocator (EEL) que constrói o indice da expertise dos grupos baseando-se efl documentos produzidos pelos membros destes grupos. Também é apresentado ContactFinder que utiliza agentes inteligentes para monitorar discussões relacionadas um determinado assunto em e-mails identificando os usuários que fazem uso do supor enico da organização. E tambem apresentado o trabalho de Schwartz \& Wood que ecrico algoritmos heurísticos para identificar através de mensagens de e-mail interesse tiliza algortos entre grupos de pessoas. O sistema DEMOIR permite encontrar peritos compartilhados ento domínio baseado em uma base de dados chamada trailbase, que em um deternunas visitadas e nas keywords utilizadas para pesquisa destas páginas. McDonald e possuem determinado conhecimento e propõe a arquitetura de de identificar pessoa quartise Recommender. Para realizar a identificação desta uma ferramenta deno princípio que trocas narrativas pessoas, McDonalise e competência. São então definidas regras heurísticas para realizar demonstram expertise e competencia. São enta defture, tais como: quem alterou por tais identificações num ambiente de projeto de software,

Cabe ainda citar Agostini e outros (2003), que descrevem o sistema MILK, que ontologias para encontrar projetos similares, pessoas que tem interesses similares ou usa ontologias parares as peciadas a conceitos de uma ontologia de documentos similares. As esta associação recebe um grau, indicando acordo com suas atividades no sistema e esta associação recebe um grau, indicando

Os sistemas descritos procuram, na maioria dos casos, monitorar as ações dos membros de uma organização, o que sem dúvida nenhuma pode ser útil. Ocorre que em muitas situações este monitoramento não pode ser feito, já que em muitas organizações nem toda atividade realizada está registrada e em alguns casos este monitoramento pode iem da navegação do usuário). Além disso, no momento em que um novo membro ingressa na organização não é possível determinar suas áreas de interesse.

\section{Sistema para Identificação de Expertise em Currículos}

No presente trabalho, a definição automática do perfil do usuário é feita através da extração de informações em textos, obtidas em um arquivo XML gerado a partir das ferramentas disponíveis no Currículo Lattes, componente que integra a Plataforma Lattes desenvolvida pelo CNPq.

O Currículo Lattes foi escolhido por ser um padrão já estabelecido (especialmente na comunidade acadêmica), por ser uma fonte completa de informação contendo dados sobre formação acadêmica, titulações, artigos escritos e área de trabalho. Além disto, pesou o fato de ser possível gerar um arquivo XML com os dados do currículo.

Na ferramenta, em um primeiro momento é feita à extração automática de todas as informações existentes no arquivo gerado pela plataforma Lattes. Levando-se em consideração o fato de se tratar de uma ferramenta que rodará em ambiente Web e que a linguagem utilizada no contexto do projeto SisRecCol (descrito na introdução) é PHP, foi necessário buscar recursos oferecidos por esta linguagem para realizar a extração dos dados.

Foram utilizados os métodos oferecidos pela classe XPath (tp://sourceforge.net/projects/phpxpath/), que manipula arquivos XML. 
Uma vez extraídos as informações do currículo, estes dados são passados uma ferramenta que aplica uma técnica de Text Mining. A técnica utilizada cons para comparar as palavras extraídas do currículo Lattes com as palavras presentes em ontologia de domínio. Detalhes relacionados ao método são fornecidos na seção 3.1 .

Terminado o processo é apresentado o conjunto de conceitos (áreas) aos q usuário está relacionado. Estes conceitos estão presentes na ontologia e apontam assuntos ou temas que são representativos dentro de uma organização.

\subsection{A Técnica de Text Mining}

Para a identificação das áreas de competência e das expertises é utilizada neste trabalh uma técnica de Text Mining que trabalha examinando o conteúdo de todo o currículo.

Conforme descrito na seção 3 , essa identificação é feita pela comparacão de termos que aparecem no currículo, com termos que estão relacionados aos conceito presentes na ontologia do sistema.

Neste sistema esta sendo utilizado um tipo de classificação baseado em técnic probabilísticas, que foi apresentado em Loh e outros (2000). Com base nos algoritmo de Rocchio e Bayes (Rocchio, 1966; Ragas \& Koster, 1998; Lewis, 1998), é usado um algoritmo que representa textos e conceitos através de vetores. Estes vetores são compostos por uma coleção de termos com um peso associado a cada termo. No caso dos textos que compõe os currículos, o peso de cada termo é dado pelo cálculo da Freqüência Relativa de cada termo no currículo. A Freqüência Relativa é o número de ocorrências da palavra no currículo, dividido pelo número total de palavras presentes no currículo. O peso de um termo representa a probabilidade deste termo indicar um determinado conceito presente na ontologia. Na montagem dos vetores são ignorados os termos que não tem muita relevância na identificação dos conceitos ou que aparecem com muita freqüência, como as preposições, artigos e etc. Estes termos são chamados de stopwords.

Utilizando uma função de similaridade que calcula a distância entre dois vetores, - método avalia a similaridade entre um vetor (currículo) e um conceito presente na ontologia. A função de similaridade multiplica os pesos dos termos que estão presentes nos dois vetores (currículo e ontologia), sendo que a soma destes produtos, restringida a 1, é o grau de similaridade existente entre o currículo e o conceito existente na ontologia. Este grau determina qual a probabilidade do conceito estar presente no currículo, ou seja, qual a probabilidade de um membro ter afinidade com determinadas áreas representada pelos conceitos existentes na ontologia.

\subsection{A Ontologia}

A ferramenta utiliza uma ontologia de domínio para classificar currículos, realizando assim a identificação das expertises e traçando o perfil dos usuários.

Uma ontologia é uma definição formal e explícita de conceitos (classes ou categorias) e seus atributos e relações (Noy e McGuinnes, 2002). Uma ontologia do domínio - domain ontology é uma descrição de "coisas" que existem ou podem existit em um domínio (Sowa, 2002).
No sistena proposto, a ontologia é implementada cer fruica, contendo um conjunto de conceitos. Cada conceito tem associado a si uma hierárquica, conten seus respectivos pesos, que ajudam e identificar o conceito presente nos lista de termos a determinam a probabilidade deste term

Os pesos associados aos termo pode um conceito diferentes, e um conceito pode ser aparecer em mais diferentes termos.

identificado por diferentes termos.

$\mathrm{Na}$ versão atual, somente uma ontologia para a Con (www.acm.org), existe ssificação da $A C M$ - Association for Compution ser adicionadas. É importante ressaltar class no sistema. En área de aplicação torna-se necessário construir uma ontologia no que para cada nova áre interesses de cada organizaça, possibilitando assim a sistema que se adapte aos interesses do conhecimento.

identificação das com

\section{Experimentos}

Antes de avaliar a ferramenta com o uso de currículos, foram feitos testes sobre textos

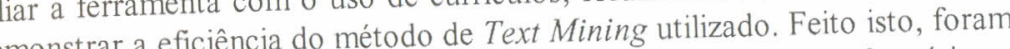
de forma a demonstrar a eficiencia de informática. feitos testes sobre um conjunto de curriculos obtidos junto a professores de informatica.

Os testes procuraram fazer a identificação de áreas da ontologia relacionadas textos e aos currículos.

\subsection{Avaliação do método de Text Mining}

Foram selecionados entre as áreas da Computação 15 artigos científicos presentes na (westes rearchindex.com). Destes artigos foram , entrada no módulo de Text capturado os resumos (abstracts), e então sinulado uma entrada método empregado. Mining com a finalidade de saber qual a porcentagen de acerto do metodo empregado. Foram avaliados tanto os resumos inteiros de cada artigo, como rase a frase pertencentes aos mesmos resumos. Foram consideradas certas as respostas que correspondiam ao conceito em que este artigo estava indexado na Biblioteca Digital ResearchIndex. Por exemplo, se o documento pertencia na base de dados à classe "Artificial Intelligence", só seria computado como certo se o módulo de Text Mining "Antificial Intelligence", só seria conputado como certo se 0 módulo de Text Mining identificasse o conceito "Artificial Intelligence". Foram avaliadas 78 frases obtidas partir dos resumos. Os resultados são apresentados na tabela 1. Já se esperava em textos maiores, meles, como os resumos inteiros, men número de características (termos) presentes.

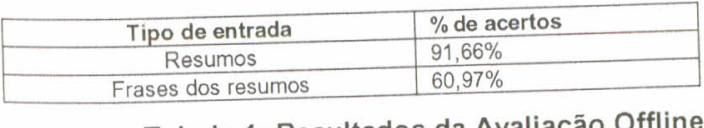

Tabela 1: Resultados da Avaliação Offline

\subsection{Avaliação da identificação de expertise sobre Currículos}

Nos experimentos descritos nesta seção procurou-se demonstrar que além de ser possivel reconhecer os interesses atuais de um usuário através da extração das 
informações do currículo Lattes, também é possível reconhecer as mudanças de inte ao longo dos anos.

Nos testes iniciais, foram analisados integralmente os currículos de professo da Escola de Informática da UCPel, sendo considerada correta a identificação se primeiro conceito retornado do módulo de Text Mining estivesse de acordo com principal área de interesse do usuário. Os experimentos revelaram uma porcentagem a torno de $60 \%$ de acertos, isto sem qualquer tipo de filtragem das informações contid nos currículos, ou seja, sendo analisadas todas as informações mesmo algumas pouco relevantes.

É importante ressaltar que um determinado usuário poderá ter afinidade con diferentes áreas. Neste sentido foi utilizado para os experimentos em currículos um limi de corte, onde foi estabelecido que conceitos retornados do módulo de Text Mining com peso abaixo de $0,001 \ldots$, seriam descartados por acreditar-se que esses são irrelevantes para análises da expertise do usuário. Este limiar pode ser alterado sempre que for necessário.

Foram também feitos testes sobre porções do currículo. Assim, foram escolhidas informações referentes a determinados anos para serem analisados. A saber, foram avaliadas publicações, orientações de graduação, apresentações e etc, nos periodos de 1999 e 2003. A idéia de avaliar períodos de tempo do currículo é tentar verificar possíveis mudanças nas áreas de interesse dos usuários.

Alguns dos resultados que foram obtidos a partir do módulo de Text Mining do experimento relativo a um dos currículos avaliados são apresentados nas figuras 1 e 2, como forma de ilustrar os testes. Nestas figuras são mostrados os dois primeiros conceitos da ontologia identificados como sendo áreas de atuação do usuário.

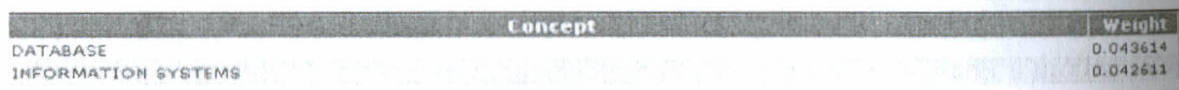

Figura 1: Resultados da Avaliação do currículo no Formato Lattes de 1999

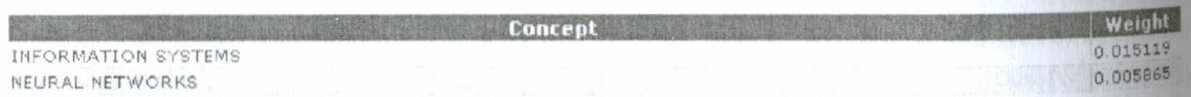

MEUR.AL NETWORKS

Figura 2: Resultados da Avaliação do currículo no Formato Lattes de 2003

A partir do teste realizado foi possível identificar as áreas de competência do usuário, assim como as mudanças de suas expertises em períodos de tempo. Pôde-se constatar que o usuário conforme a figura 1 teve seu perfil no ano de 1999 , relacionado a "Database", e conforme a figura 2, no ano de 2003, relacionado ao conceito "Information Systems", sendo assim constatada uma leve mudança na área de interesse do usuário no referido período de tempo.

Esta identificação em organizações pode significar um diferencial em relação às outras, uma vez que a partir da definição precisa do perfil de seus funcionários é possivel avaliar a real necessidade de treinamentos ou uma possível mudança de domínio da empresa utilizando melhor o conhecimento existente no seu quadro de funcionários ou ainda a necessidade de aquisição de novas competências para atender as suas necessidades. último, um fator importante a ser considerado nos resultados é o fato de que por ultino a análise correspondem aos termos presentes em funcão disto, a dados relevantos exemplo, nos títulos dos trabalhos publicados. do curriculo, de acerto é diminuída em comparação a teste pssibilidade de acrito na seção 5.1 . como o resur feitos também experimentos analisando as inforalizados para identificar Foram eltacões de forma separada. Estes testes frabalhos como orientador.

as orientaç de uma relação entre as publicações e os trabalhos como neção 5.1. Os

Os testes foram realizados com a metodologia descrita a ́a que o usuário Os resultados indicaram qué géne porém, torná-la explícita facilita até mesmo a resulta. Esta relação é quase obvia, porem, toricando exatamente a área que cada definição de um orientador por parte de um

professor está se dedicando no momento.

\section{Conclusões e Trabalhos futuros}

Este trabalho apresentou uma ferramenta que auxilia em processos de Gestão de expertises de pessoas através da análise das Competencias, permitindo identificar informações extraídas do

desenvolvido pelo $\mathrm{CNPq}$ que maior bem é o conhecimento dos seus mato conhecimento e funcionários, existindo, porém uma certa dificuldade para cata

em determinar quem são especialistas em determinadas áreas.

Isto faz da análise dos currículos dos membros de uma orgável apontar em possível aplicã deste trabalho. Com esta ferramenta, podera ser possiel apontar em possivelaplicacĩo deste tempetências presentes aos curriculos dos membros organização. cos primeiros testes, porém, plataforma Lattes, se mostrou bastante eficiente dura

ainda existem alguns aperfeiçoamentos a serem feitos.

Uma das limitações da aplicação da ferramenta pode estar no uso do curriculo nó formato Lattes, que vem sendo largamente utilizado no ambiente acadénico, mas descritos adotado com freqüência em outros ambientes. No entanto, os métodos. Neste caso, a poderão ser utilizados em currículos disponibilizados em outros formatos. Ne partes do principal limitação irá talvez residir na dificuldade de extrair informaçoes de partes do currículo. a especial, sendo necessário pensar em formas de facilitar a sua construção. Na versão atual, parte do processo de form a das informações relativas a determinados periodos duro. 
Versões futuras também levarão em conta o fato de que termos que aparecem determinadas partes do currículo, deverão ter maior peso na identificação do perfil usuário. No caso da estrutura do currículo Lattes, termos que aparecem no elemento áreas-de-atuação devem provavelmente ter maior peso do que termos que apareco relacionados ao elemento participação-em-eventos-congressos.

A análise temporal torna-se importante, pois mesmo que um determinado usuário seja reconhecido como especialista em determinada área, deve-se levar em consideraça o período em que trabalhou nesta área. Evitando que uma pessoa que tenha mudado de competência (ou de interesses) com o passar dos anos, seja identificado como especialista.

\section{Agradecimentos}

O presente trabalho foi realizado com o apoio do $\mathrm{CNPq}$, uma entidade do Governo Brasileiro voltada ao desenvolvimento científico e tecnológico.

\section{Referências}

AGOSTINI, A. et al. (2003) Stimulating knowledge discovery and sharing. Proceedings of the International ACM SIGGROUP Conference on Supporting Group Work. Sanibel Island, USA, p.248-257.

CNPq, Conselho Nacional de Pesquisa e qualidade. Disponível pela URL: http://lattes.cnpq.br/

DAVENPORT, T. H. e PRUZAC, L. (1997) "Working knowledge - How organizations managewhat they know", Harvard Business School Press, Harvard.

LEWIS, D. D. (1998) "Naive (bayes) at forty: the independence assumption in information Retrieval", in: Proc. European Conference on Machine Learning, Lecture Notes in Computer Science, v.1398, Springer, Berlin, p. 4-15.

LOH, S. ; WIVES, L. K.; OLIVEIR, J. P. M. (2000) "Concept-based knowledge discovery in texts extracted from the Web", ACM SIGKDD Explorations 2 (1), p. 29 . 39.

MCDONALD, D.W. e ACKERMAN, M.S. (2000) "Expertise recommender: a flexible recommendation system and architecture" in Proc. ACM Conf. on Computer Supported Cooperative Work, Philadelphia, p.231-240

NOY N. F. e MCGUINESS, D. L. (2002) "Ontology Development 101: a guide to creating your first ontology". Disponível em http://protege.stanford.edu/publications.

ROCCHIO, J. J. (1966) "Document retrieval systems - optimization and evaluation", Ph.D. Thesis, Harvard Computation Laboratory, Harvard University, Report ISR-10 to National Science Foundation.

SOWA, J. F. (2002) "Building, sharing, and merging ontologies", AAAI Press / MIT press, pages 3-41

YIMAM-SEID, Dawit; KOBSA, Alfred. Expert Finding Systems for Organizations: Problem and Domain Analysis and the DEMOIR Approach. Journal of Organizational Computing and Electronic Commerce, v. 13, n. 1, 2003, p. 1-24. 